\title{
Case Report: Intraocular Non-Hodgkin Lymphoma
}

\author{
Ana Cabugueira*, Vanessa Lemos, Marco Dutra Medeiros, Rita Flores and Pinto Ferreira
}

Central Lisbon Hospital Center, NOVA Medical School, Universidade NOVA de Lisboa, Lisbon, Portugal

\begin{abstract}
Purpose: Primary intraocular lymphoma is a primary central nervous system lymphoma in which lymphoma cells invade the retina, vitreous, or optic nerve head without concomitant central nervous system involvement. The aim of this presentation is to report a case of primary intraocular non-Hodgkin lymphoma.

Methods: The authors present a case report of a 77 years-old female with painless decreased in visual acuity and floaters. Ophthalmic examination, Static computerized Perimetry, Spectral-Domain Optical Coherence Tomography, angiography, laboratory study, lumbar puncture, computed tomography scan and magnetic resonance imaging were performed.

Results: On examination, best corrected visual acuity was 20/25 in the right eye and 30/60 in the left eye. Slip lamp examination revealed anterior chamber reaction and fundoscopy showed vitritis, optic disc edema, macular edema and vasculitis in left eye. After three months of follow-up, the patient's best corrected visual acuity decreased to $20 / 60$ in right eye and light perception in left eye. Fundoscopy revealed vitritis, optic disc edema, macular edema and vasculitis in both eyes. A granular pattern, leakage from retinal vessels and optic disc were observed in fluorescein angiography. Infectious and inflammatory etiologies were excluded. Pars plana vitrectomy and retinal biopsy were performed in left eye. Cytology evaluation revealed atypical lymphoid cells with large nuclei, prominent nucleoli and basophilic cytoplasm, and confirmed the diagnosis of intraocular lymphoma.
\end{abstract}

Conclusion: Intraocular Lymphomas are rare malignancies that display a wide array of clinical manifestation, therefore diagnosis can be challenging. It requires a high degree of clinical suspicion and differential diagnosis includes infectious and non-infectious etiologies.

Keywords: Primary intraocular lymphoma; Central nervous system; Fundoscopy; Vasculitis

\section{Introduction}

Primary Intraocular Lymphoma (PIOL) is a rare malignancy of primary Central Nervous System (CNS) without concomitant intracranial involvement. If there was vitreoretinal lymphoma with CNS disease, this is classified as Primary Central Nervous System Lymphoma (PCNSL) [1]. In PIOL, usually, lymphoma cells invade the retina, vitreous, or optic nerve head whereas secondary intraocular lymphoma predominantly infiltrates uveal tract, particularly the choroid. PIOL, usually, presents as a diffuse, large B-cell non-Hodgkin's lymphoma. The etiology of PIOL and PCNSL are still unknown $[1,2]$. The average age in immunocompetent patients is the late $50 \mathrm{~s}$ and 60 s and the rate appears to be increasing dramatically, especially in immunocompromised patients [3].

The diagnosis is difficult and often delay, because it can present with a wide variety of manifestations, that can mimic many ocular conditions [3]. Classic presentation is painless visual acuity decline with floaters and chronic uveitis poorly responsive to corticosteroids [4]. On Slit-lamp examination, anterior chamber cells are presented up to $75 \%$ of cases. The most frequent posterior segment signs are vitritis and classic lesions, such large, multifocal, cream or yellow subretinal infiltrates. This lesions are considered pathogmonic of this disease. After the resolution of this lesions a leopard-spot apperence may be seen. Other manifestations are multiple white dots, retinal infiltration, retinal vasculitis, vein and arterial occlusion, fundus flavimaculatuslike, exsudative retinal detachment, choroidal involvement, optic disc invasion [5].

The diagnostic approach includes neurological evaluation, such Computerized Tomography (CT), Magnetic Resonance Imaging (MRI), Lumbar Pucntion (LP) and vitreous or chorioretinal biopsy.

$60-80 \%$ of patients with PIOL develop cerebral lymphoma and systemic spread is rare, occurring in only 6 to $8 \%$ of cases. The average time separating the onset of ocular symptoms and CNS disease is 29 months [3]. It requires a high degree of clinical suspicion and differential diagnosis includes infectious and non-infectious etiologies. 6 Optimal treatment for PIOL and PCNSL with ocular involvement is controversial and has yet to be defined. The aim of our study was to describe a case of a rare malignancy as PIOL and the diagnostic work-up.

\section{Case Report}

The authors describe a case of a 77 years-old, caucasian female, presented with painless decreased visual acuity and floaters in her Left Eye (LE) with five days of evolution. In the first visit, Best Corrected Visual Acuity (BCVA) was 20/25 on the Right Eye (RE) and 20/60 on the LE.

Slit-lamp examination revealed deep anterior chamber with + 2 tyndall. Intraocular pression was $16 \mathrm{mmHg}$ and $15 \mathrm{mmHg}$ in $\mathrm{RE}$ and LE, respectively. Fundus examination showed vitritis, optic disc swollen, macular edema and vacuities in the LE (Figure 1). The patient denied other neurological complains.

Systemic and laboratory evaluations including complete blood count, erythrocyte sedimentation rate, C-reactive protein, tuberculin skin test, angiotensin converting enzyme,urine analysis, chest X-ray,

*Corresponding author: Ana Cabugueira, Central Lisbon Hospital Center, NOVA Medical School, Universidade NOVA de Lisboa, Lisbon; Portugal, Tel: +351 21371 5600; E-mail: anacabugueira@hotmail.com

Received April 25, 2015; Accepted August 06, 2015; Published August 13, 2015

Citation: Cabugueira A, Lemos V, Medeiros MD, Flores R, Ferreira P (2015) Case Report: Intraocular Non-Hodgkin Lymphoma. J Clin Case Rep 5: 579. doi:10.4172/2165-7920.1000579

Copyright: (c) 2015 Cabugueira A, et al. This is an open-access article distributed under the terms of the Creative Commons Attribution License, which permits unrestricted use, distribution, and reproduction in any medium, provided the original author and source are credited. 
HLA-B5, HLA-B51 and HLA-B27 were negative. Infectious and inflammatory etiologies were excluded and CT brain scans were normal. Topical treatment with fluorometholone $0.1 \%$ drops every 4 hours, tropicamide $1 \%$ every 8 hours was started with no clinical improvement.

After three months, visual acuity in both eyes (OU) declined to 20/60 in right eye (RE) and light perception in LE. On ophthalmic examination, anterior chamber was deep and quite, and posterior segment revealed vitritis, optic disc swollen, macular edema and vasculitis in OU. A granular pattern leakage from retinal vessels and optic disc were observed in FA (Figure 2) [6-8].

Neurological studies were performed. MRI did not show alterations and cerebrospinal fluid didn't revealed significative alterations. Pars plana vitrectomy and retinal biopsy were performed in LE. Cytology evaluation revealed atypical lymphoid cells with large nuclei, prominent nucleoli and basophilic cytoplasm, and confirmed a diagnosis of intraocular lymphoma (Figure 3). Despite of treatment with intravitreal and intrathecal methotrexate in LE, patient had other comorbilities and died one week later.

\section{Discussion}

PIOL, often masquerades as intraocular inflammation and is easily misdiagnosed so it represents a diagnostic and therapeutic challenge for ophthalmologists and pathologists. Patients, usually present blurred vision and floaters and, less commonly, photophobia and ocular pain. Typical signs include cells in the anterior chamber and clumps or sheets of cells in the vitreous [5]. Lymphocytic cells invade sub-RPE, above the Bruch's and on fundoscopy different images can illustrate characteristic patterns. Fundus autofluorescence showed bright hyperfluorescent spots corresponding to the sub-RPE infiltrates, and hypofluorescent areas corresponding to RPE atrophy, where presumably, the tumor cells were previously. FA illustrates RPE disturbances as granular, mottling, and late staining patterns. Clusters of round, hyper- or hypo-fluorescent spots reflect RPE abnormalities. Ocular coherence tomography shows nodular hyperreflective lesions at the RPE level [5].

The diagnostic approach of patients with primary intraocular lymphoma includes complete ophthalmologic examination, neurological evaluation, neuroradiologic imaging of the CNS with contrast-enhanced CT or MRI, CSF cytology, and vitreous or

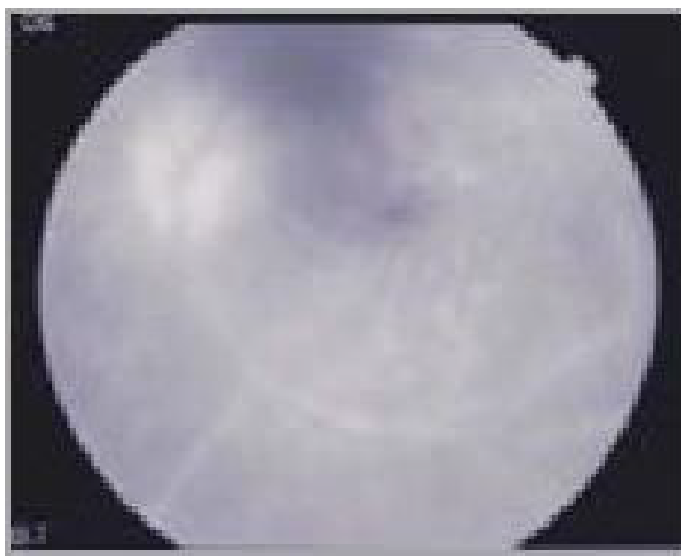

Figure 1: Angiography of the LE.

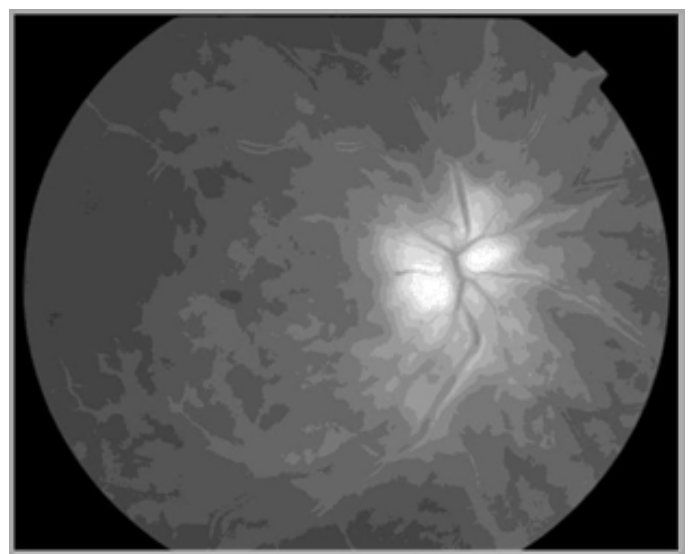

Figure 2: Angiography of the RE.

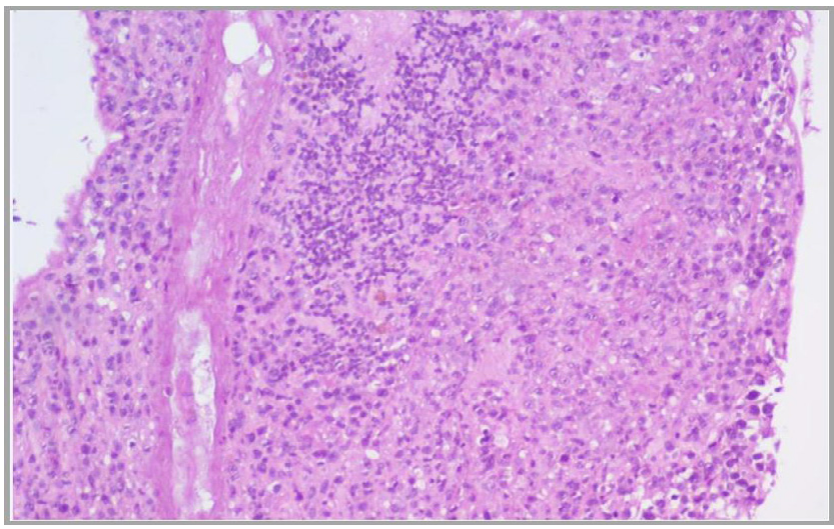

Figure 3: Cytopathologic features of the LE.

chorioretinal biopsy. The differential diagnosis includes infectious, inflammatory, and other neoplastic processes $[7,9,10]$.

Infectious etiologies include endophthalmitis secondary to bacterial, viral, fungal, or parasitic infections. Inflammatory etiologies include uveitis-glaucoma-hyphema syndrome, sympathetic ophthalmia, sarcoidosis, pars planitis, multiple sclerosis, rheumatoid arthritis, birdshot chorioretinopathy, Behcet disease, Vogt-KoyanagiHarada disease, sterile endophthalmitis, multifocal choroiditis, and acute posterior multifocal placoid pigment epitheliopathy $[5,6]$. The definitive diagnosis requires histological analysis, that could be achieve by lymphocytic cells CSF, vitreous, retina or brain.

Regarding treatment of isolated PIOL, the current trend propose local treatment as first line and early diagnosis. But there was a high degree of morbidity and mortality and further studies are needed [1,7]. In summary, clinical manifestation, imaging findings, flow cytometry, are helpful adjuncts for correct diagnosis, although the definitive diagnosis relies on the identification of lymphoma cells in the retina, vitreous, and/or optic nerve.

\section{References}

1. Hanson J, Alexandru D, Bota D (2013) The evaluation and treatment of primary intraocular lymphoma. Journal of Cancer therapeutics and research 2: 15.

2. Faia L, Chan C (2013) Primary Intraocular Lymphoma. Arch Pathol Lab Med 133: $1228-1232$ 
Citation: Cabugueira A, Lemos V, Medeiros MD, Flores R, Ferreira P (2015) Case Report: Intraocular Non-Hodgkin Lymphoma. J Clin Case Rep 5: 579. doi:10.4172/2165-7920.1000579

3. Chan C, Wallace D (2004) Intraocular Lymphoma: Update on Diagnosis and Management. Cancer control 5: 285-295.

4. Hoffman P, McKelvie P, Hall A (2003) Intraocular lymphoma: a series of 14 patients with clinicopathological features and treatment outcomes. Eye 17: 513-521.

5. Saggo M, Mehta H, Cohen V, Amin S, Plowman P, et al. (2014) Primary Intraocular Lymphoma. Survey ophthalmology 59: 503-516.

6. Sen H, Hoang B, Nussenblatt R (2009) Primary intraocular Lymphoma: Diagnosis and Differential Diagnosis. Ocul Immunol Inflamm 17: 133-141.
7. Kristoph J, Eckhard T, Abrey L, Neuwelt E, Korfel A (2007) Diagnostic and management of primary intraocular lymphoma: an update. Clinical Ophthalmology 1: 247-258.

8. Karimi M, Soheleilian M, Kanavi M (2011) Bilateral Primary Intraocular Lymphoma. 6: 344-347.

9. Choi J, Kaflan C, Foster C (2006) Primary intraocular Lymphoma: A review. Semin Ophthalmol 21: 125-133.

10. Char D, Kemlitz A, Miller T (2005) Intraocular biopsy. Ophthalmol Clin N Am 18: $177-85$. 